\title{
Video Article \\ Automated Visual Cognitive Tasks for Recording Neural Activity Using a Floor Projection Maze
}

\author{
Tara K. Jacobson* ${ }^{1}$, Jonathan W. Ho* ${ }^{1}$, Brendon W. Kent ${ }^{1}$, Fang-Chi Yang ${ }^{1}$, Rebecca D. Burwell ${ }^{1,2}$ \\ ${ }^{1}$ Department of Cognitive, Linguistic \& Psychological Sciences, Brown University \\ ${ }^{2}$ Department of Neuroscience, Brown University \\ * These authors contributed equally
}

Correspondence to: Rebecca D. Burwell at rebecca_burwell@brown.edu

URL: https://www.jove.com/video/51316

DOI: doi:10.3791/51316

Keywords: Neurobiology, Issue 84, Rat behavioral tasks, visual discrimination, chronic electrophysiological recordings, Floor Projection Maze, neuropsychology, learning, memory

Date Published: 2/20/2014

Citation: Jacobson, T.K., Ho, J.W., Kent, B.W., Yang, F.C., Burwell, R.D. Automated Visual Cognitive Tasks for Recording Neural Activity Using a Floor Projection Maze. J. Vis. Exp. (84), e51316, doi:10.3791/51316 (2014).

\section{Abstract}

Neuropsychological tasks used in primates to investigate mechanisms of learning and memory are typically visually guided cognitive tasks. We have developed visual cognitive tasks for rats using the Floor Projection Maze ${ }^{1,2}$ that are optimized for visual abilities of rats permitting stronger comparisons of experimental findings with other species.

In order to investigate neural correlates of learning and memory, we have integrated electrophysiological recordings into fully automated cognitive tasks on the Floor Projection Maze ${ }^{1,2}$. Behavioral software interfaced with an animal tracking system allows monitoring of the animal's behavior with precise control of image presentation and reward contingencies for better trained animals. Integration with an in vivo electrophysiological recording system enables examination of behavioral correlates of neural activity at selected epochs of a given cognitive task

We describe protocols for a model system that combines automated visual presentation of information to rodents and intracranial reward with electrophysiological approaches. Our model system offers a sophisticated set of tools as a framework for other cognitive tasks to better isolate and identify specific mechanisms contributing to particular cognitive processes.

\section{Video Link}

The video component of this article can be found at https://www.jove.com/video/51316/

\section{Introduction}

Visual tasks are commonly used in human and monkey studies to examine mechanisms underlying learning and memory. Rodent models, however, are more readily accessible to researchers for larger scale, better controlled studies, and have the added advantage of permitting more invasive electrophysiological techniques. In combination with other approaches, such as genetic manipulations, electrophysiological recordings in freely moving rats provide a useful model for precisely targeting mechanisms and circuits underlying cognitive processes. Primate visual tasks require the subjects to direct the gaze to visual stimuli on a monitor, whereas rat tasks require the subjects to interact with an environment. The Floor Projection Maze utilizes the natural tendency of rats to actively explore the environment while simultaneously attending to visual stimuli.

Cognitive tasks using touchscreen apparatuses designed specifically for rodents have allowed better translation of findings from rodent models to humans ${ }^{3}$. Touchscreen tasks are typically carried out in a chamber with two dimensional visual stimuli presented vertically onto walls ${ }^{3-7}$. These touchscreen tasks require the subject to rear towards the target visual stimulus and break an infrared photobeam or press on a pressure pad to register its response. Anatomical and behavioral evidence, however, suggest that rats process visual information in the lower visual hemifield more effectively for guiding behavior ${ }^{8-10}$. Our lab has developed cognitive tasks utilizing the Floor Projection Maze ${ }^{2}$ in which two-dimensional visual stimuli are back projected onto the floor of the test arena. In the Floor Projection Maze rats can be tracked when performing tasks in a large open arena compared with touchscreen apparatus. Thus, spatial information in the recorded neural activity can be obtained in addition to neural correlates of visual information and decision-making.

We deliver intracranial stimulation (ICS) to the medial forebrain bundle (MFB) as a reward ${ }^{11}$. This method of reward delivery has advantages over food and drink rewards. Food and drink rewards can lead to satiation, even in food-deprived rats, limiting the number of trials an animal will perform and potentially slowing the training process. ICS delivers instant reward providing immediate feedback on task performance. The immediate reward results in faster shaping and acquisition and substantially reduces the duration of training protocols. Moreover, a larger number of trials can be completed in a session, increasing the amount of data collected and resulting in a more reliable sample of task-related behavior. 
Using the Floor Projection Maze, we will describe a general protocol to shape the behavior of rats to perform complex cognitive tasks. This general protocol provides a framework for training rats across a variety of tasks currently employed for recording neural correlates of attention and visual discrimination ${ }^{1}$. Thus, the Floor Projection Maze is optimized for visual abilities of rats and permits stronger comparisons with visual tasks in humans and nonhuman primates.

\section{Protocol}

All procedures were in accordance with Brown University Institutional Animal Care and Use Committee guidelines.

\section{Systems Overview}

A video tracking system interacts with a behavioral control system to monitor the rat's progress in a given task, evaluate target behaviors, control stimulus presentation and deliver rewards based on the rat's progress. An vivo electrophysiological recording system collects neural data for event related analyses. (Figure 1A).

1. The Floor Projection Maze

1. The Floor Projection Maze ${ }^{2}$ is an open field without walls $(147.3 \mathrm{~cm} \times 111.8 \mathrm{~cm})$ with a clear colorless Plexiglas floor $(0.6 \mathrm{~cm}$ thick). The underside of the floor is a unity gain Dual Vision Fabric screen that is stretched over a second rectangle of Plexiglas pieces (147.3 $\mathrm{cm} \times 111.8 \mathrm{~cm} \times 1.25 \mathrm{~cm}$ ) for rear projection using a short throw projector.

2. Connect the projector to the video card in the behavioral system PC. Hold the Plexiglas, screen fabric, and projector using an anodized aluminum frame.

3. Install an overhead anodized frame to mount an overhead camera and a pulley system to interface the ICS and headstage tethers to the rat.

Note: Connect the frame holding the Floor Projection Maze and overhead frame to ensure electrical continuity and ground to the preamplifier.

2. Video tracking

1. Record, track and analyze videos in real time with a single overhead camera described in step 1.2.2.

2. Mount an overhead camera (standard VGA, $80 \mathrm{fps}$ ) onto an overhead anodized aluminum frame to monitor the rat

3. Use either light emitting diodes (LEDs) attached to the rat's headstage to monitor the position of the rat, or track the centroid of the contour of the rat with an automated tracking system.

4. Use the CinePlex Basic Behavior module to analyze the position data online and also save the data file for further offline analysis. Note: We use the CinePlex Behavioral Research System 3.4.1 for recording, tracking and analyzing the rat's position. The CinePlex Tracking module is used for tracking the rat. For effective tracking of the animal's progress, use the CinePlex Basic Behavior module to create zones in two-dimensional space at relevant positions of the maze that have significance in the task. Zones can be combined into sequences, and CinePlex logical events (such as entries into zones and fulfillment of the sequences) are assigned such that if the rat satisfies the criteria for the event, that event becomes true. Assign events with digital outputs and interface via a superport input card to the behavioral system.

3. A fully automated behavioral control system

1. Behavioral test arena: Use an appropriate shaped test arena for the behavioral task on the Floor Projection Maze. Construct the arena using matte white acrylic and place it directly on the Floor Projection Maze.

Note: Dimensions can be customized for the behavioral task used. Walls are typically $45-50 \mathrm{~cm}$ in height. Here we use double-sided test arenas (bowtie maze ${ }^{12}$ ) to maximize the number of trials performed in a session (Figure 1B for dimensions of the bowtie maze). The test arena has four designated areas defined in the tracking software: East Image area, West Image Area, an East Trial Ready Area, and a West Trial Ready Area (Figure 1C).

2. Behavioral system: A behavioral interface system controlled by MED-PC IV software runs programs written in-house in MEDstate Notation (see Appendix). Use the events from CinePlex behavioral system to track a rat's progress during the task, control image presentation, deliver audio cues and deliver reward.

1. Interface the superport output card to Omniplex digital input to acquire time stamped behavioral event occurrences in the PlexControl software environment for offline analyses. Issue auditory cues using a programmable audio generator. Note: Behavioral events from the apparatus presented here can operate third party equipment (e.g. laser for optogenetic stimulation) via a data transfer board if required.

2. House the superport output card, superport input card and programmable audio generator in a tabletop interface cabinet. Interface the cards with a DIG-704PCI-2 PCI card installed in a personal computer (PC) using a DIG-700F Decoder card installed in the tabletop cabinet.

3. Deliver bipolar square wave ICS to the rat using a programmable intracranial self stimulation stimulator. Connect the stimulator unit to the PC via a PHM152 COM card. Recommended ICS parameters: Pulse 1 and 2 duration: $500 \mu s e c$; Delay between Pulse 1 and 2: $500 \mu \mathrm{sec}$; Frequency: $100 \mathrm{~Hz}$.

Note: ICS delivery is automated during the task but can be manually delivered using a programmable button box interfaced with a SmartCtrl module.

4. Neural data acquisition system

1. Ensure the Floor Projection Maze frame and ICS system is grounded to the preamplifier of the Omniplex Neural Data Acquisition System to minimize electrical noise in the neural recordings. 
2. Simultaneously collect neural data, positional coordinates from CinePlex and behavioral event flags from the behavioral system in the data file for offline analyses.

\section{Animal Preparation}

1. Animals

1. Obtain naïve 22 day old male Long-Evans rats.

2. Pair house the rats and allow them to acclimatize to the vivarium for one week.

3. Handle rats daily for $\sim 5 \mathrm{~min}$.

4. Once rats reach $250-275 \mathrm{~g}$, begin food schedules maintaining their body weights at $85-90 \%$ of their free feeding weight. Target weights are increased by $10 \mathrm{~g} / \mathrm{month}$ until rats reach $350 \mathrm{~g}$.

5. Single house the rats and continue the food schedule for at least a week before commencing surgery to implant stimulation and recording electrodes.

2. Surgical implantation

Carry out surgical procedures under standard aseptic conditions and according to institutional and regulatory guidelines.

1. Anesthetize rats with isoflurane

2. Make an incision in the scalp to reveal the skull for cleaning and identification of bregma and lambda.

3. Make craniotomies at the relevant coordinates.

4. Affix skull anchor screws.

5. Lower the tip of an ICS electrode into the MFB using the following coordinates: antero-posterior, $-2.7 \mathrm{~mm}$ from bregma; lateral, \pm 1.8 $\mathrm{mm}$; dorsoventral, $-8.5 \mathrm{~mm}$ from skull surface. A second ICS electrode can be implanted into the opposite hemisphere as a backup in case of electrode malfunction.

6. Secure the ICS electrodes to the skull and anchor screws with bone cement. Do not cement the pedestal (plastic housing on the stimulating electrode) of the electrodes.

7. Lower the recording electrodes into the site of interest and secure the device with bone cement.

8. Position the ICS pedestal away from the recording device and secure the ICS electrodes and recording devices to the anchor screws with bone cement.

9. Allow at least seven days recovery before commencing habituation to the shaping protocol.

\section{Behavioral Shaping: General Shaping Consists of Three Stages: Early, Intermediate, and Late Shaping (Figure 2).}

Note: The aim of Early and Intermediate shaping is to train the rat to maintain a stationary 'ready position' in the Ready Area for each trial, and perform numerous trials in a session. Semi-automate behavioral shaping so training can be adapted to a rat's individual learning rates. Once a rat is maintaining a successful 'ready position', proceed to task specific shaping (Late shaping) with the aim of transferring the rat onto a fully automated protocol for precise and unbiased behavioral control (Figure 2)

1. Early shaping (Figure 2A)

1. Day 1: Habituate the rat to the behavioral room for 10 min with the equipment switched on then return to the colony.

2. Day 2: Repeat step 1, then habituate the animal to the testing arena for $10 \mathrm{~min}$

3. Day 3: Connect the ICS and headstage tethers to the rat, and habituate the rat to the testing arena for $10 \mathrm{~min}$.

4. Day 4: Determine the lowest ICS amplitude to establish a place preference using an informal place preference conditioning protocol. Titrate Pulse 1 and 2 amplitudes. Typical amplitude values are 20-80 $\mu \mathrm{A}$.

5. From day 5: Deliver ICS reward to train the rats to associate the Ready Area and east and west image presentations with ICS reward. Continue until the rat is alternating between East and West Image Areas.

2. Intermediate shaping (Figure 2B).

1. Introduce white noise $(50 \mathrm{~dB})$ to signal the start of a trial. Turn off the white noise when the rat enters the Ready Area.

2. Automate ICS delivery for entry into the Ready Area, and for completing successful 'ready positions'. Adjust the probability of ICS delivery at entry and successful 'ready position' to reinforce behavior.

Note: Adjust the reward probabilities manually to suit individual rat's performance during shaping. Decrease reward probabilities for entry into the Ready Area and maintaining successful ready positions. Recommended final probability of reward for fulfilling successful 'ready positions is $5-10 \%$. Rats are rewarded for all correct choices.

3. Start with short 'ready position' durations (e.g. $200 \mathrm{msec})$. Gradually increase the 'ready position' durations in $100 \mathrm{msec}$ steps.

4. Turn on the white noise if the rat prematurely breaks the 'ready position' so the rat has to restart the trial.

5. Move to Late shaping when rat is holding in the 'ready position' for up to 1,200 msec.

3. Late shaping (Figure 2C)

Note: Training in Late shaping is specific for the task in which the rat will perform. Automate training in this shaping step for precise and unbiased control of all task parameters but maintain flexibility to manually deliver ICS rewards. Training protocols for two tasks are described.

1. Visual biconditional discrimination (VBCD) task: Use distinct visual stimuli to train the rats on a simple shape and luminance discriminence.

1. Begin the trial by turning on the white noise.

2. Randomly impose 'ready position' latencies of $700-1,200 \mathrm{msec}$.

3. Deliver ICS manually if required to reinforce successful 'ready positions'. 
4. Present a pair of images in the image presentation area. Pseudorandomly present the correct image on the left or right side of the Image Area.

5. Deliver ICS for a correct response and clear the floor. A correct response is registered when the rat enters the zone in which the correct image is located. On the first day of training only, issue a $75 \mathrm{~dB}$ burst of white noise as a deterrent for an incorrect response.

6. Issue a correction trial after an incorrect trial. Correction trials have identical parameters (left or right side and 'ready position' latency) as the previous incorrect trial.

7. Once the rats are able to successfully perform the simple discrimination, introduce the different floor patterns to train the biconditional discrimination rule.

8. Present a new pair of images and two distinct floor patterns to serve as the context. The correct image is conditional upon the floor pattern; e.g. the black star is correct when the floor is grey and the white circle is correct when the floor is striped (Figure 3C).

9. Pseudorandomly assign the floor pattern and the position (left or right) of the correct image for each trial ensuring that trials are counterbalanced.

10. Implement correction trials after an incorrect trial in which the trial parameters are identical to the preceding trial.

2. Visuospatial attention (VSA) task: Present illuminated white circles at defined spatial locations in the arena to train the rats to approach the spatial location of the target circle.

Note: For this task the arena has grey circles at different spatial locations in the East and West Image Areas. After a successful 'ready position', the target circle will illuminate (turn white), and the rat must approach that target location (Figure 3B).

1. Randomly impose 'ready position' latencies of $1,000-1,600$ msec on a trial by trial basis.

2. Deliver ICS manually if required to reinforce successful 'ready positions'.

3. After completion of 'ready position', randomly illuminate one of the grey circles.

4. Deliver ICS when the rat enters the zone of the illuminated circle.

5. Turn off illumination and commence the next trial on the opposite (East or West) side.

6. At the start of Late shaping, illuminate the circle until the rat approaches it or until the trial ends ( $5 \mathrm{sec}$ after illuminating the circle). Score each trial as either correct or an omission trial. For omission trials, illuminate the whole floor (white floor presented) and no reward will be available until the next trial.

7. When the rat's performance reaches $80 \%$ correct, decrease the amount of time the circle remains illuminated to $1 \mathrm{sec}$. The rat has $5 \mathrm{sec}$ to make a choice.

8. Do not reward for incorrect trials. Start the next trial.

9. If an omission trial, illuminate the floor and start a new trial.

10. At $80 \%$ correct further decrease the time the target circle is illuminated to $500 \mathrm{msec}$.

11. Choose target locations randomly before each trial. The rat will continue to have $5 \mathrm{sec}$ to make a choice before the entire floor illuminates to signal the end of that trial.

12. Do not reward for incorrect or omission trials. The rat must initiate a new trial on the opposite side of the arena.

13. Note: Additional circles may be added in each Image Area to further increase task difficulty.

\section{Representative Results}

The goal of the general shaping steps is to acclimate the rat to the testing arena, train the rat to remain in a stationary 'ready position' for the presentation of the target visual stimuli, and approach the location of the correct visual stimulus. After habituation to the testing room, arena, and tethers, Early shaping typically requires 100-150 trials until the rats are alternating between the East and West sides of the arena. During Early shaping the rats typically run through the Ready Area in center of the arena and spend most of the time exploring the perimeter of the arena (Figure 4A). Rats at the Intermediate shaping stage gradually learn to maintain a stationary 'ready position' in the Ready Area requiring 600-700 trials. At this point, animals' paths are stereotyped loops from the Ready Area to the Image Area with less time spent exploring the perimeter of the maze. However, rats are not maintaining a stationary 'ready position', indicated by the speed at which rats traverse the center Ready Area (Figure 4B).

By the end of Intermediate shaping, rats maintain a stationary 'ready position' in the Ready Area before approaching the target visual stimulus. The rat then initiates the next trial on the opposite side of the arena (Figure 4C).

The integration of the tracking software and the Neural Data Acquisition System with a behavioral control system allows for event related analyses of neural data. Drivable electrode arrays can be strategically placed to record single-unit and local field potential activity. Recordings were carried out in postrhinal cortex when rats performed the vBCD task. Perievent histograms and raster plots show that cells in postrhinal cortex respond to the onset of the target visual stimulus and to the onset of the floor pattern (Figure 5A). In posterior parietal cortex of rats performing the VSA task cells respond to the image presentation, and when the choice was made by entering the zone that defined the target image (Figure 5B). Local field potential activity in posterior parietal cortex during performance in the VSA task shows strong power in the theta range $(\sim 8 \mathrm{~Hz})$ when rats are in the 'ready position' before the image presentation (Figure 5C). 
A.

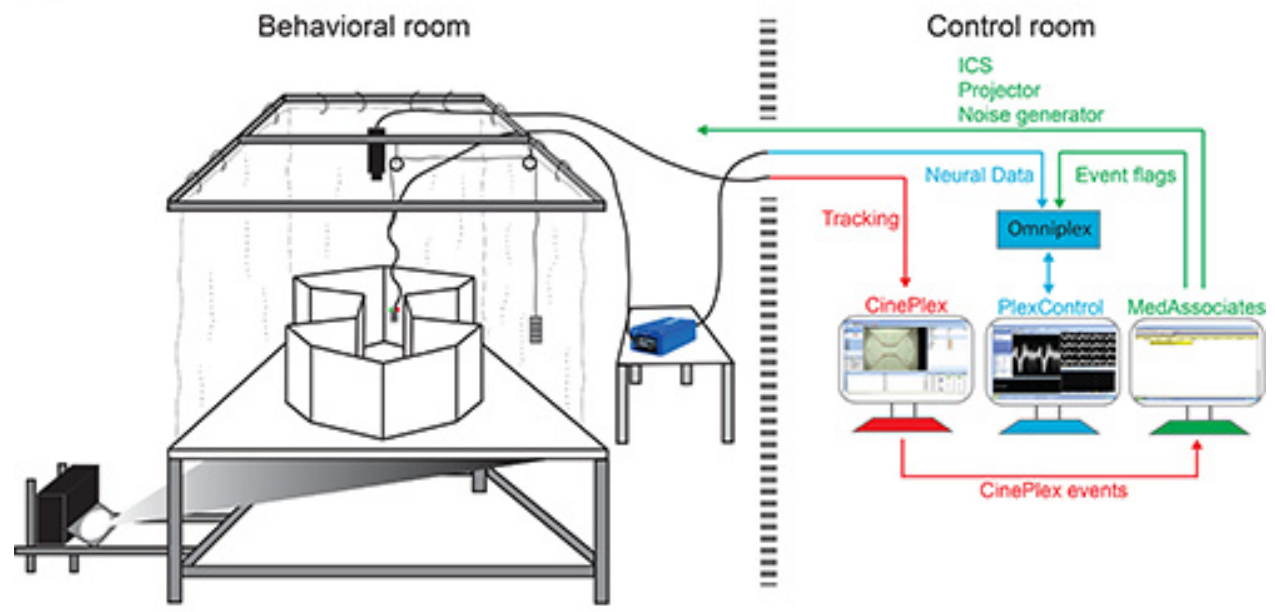

B.

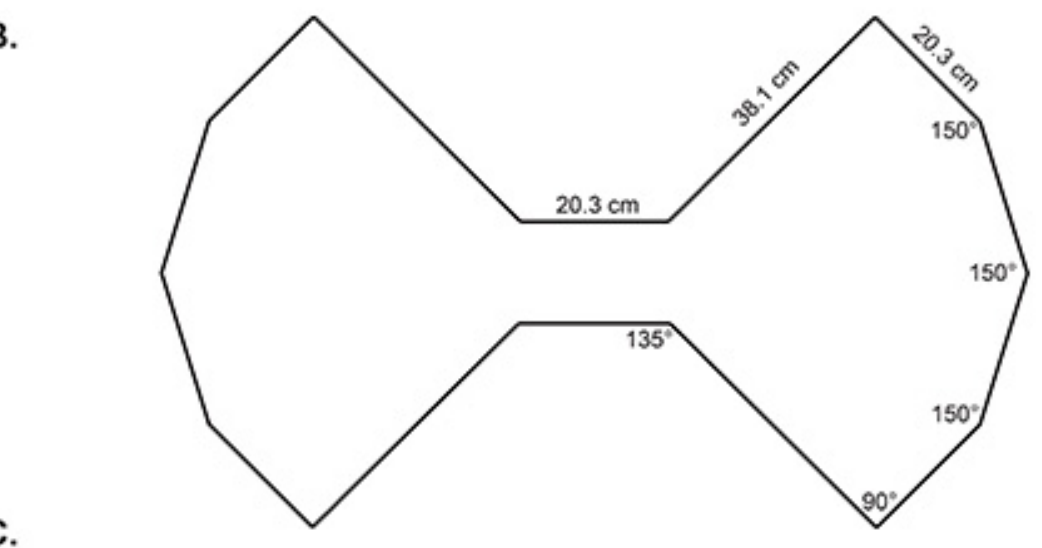

C.

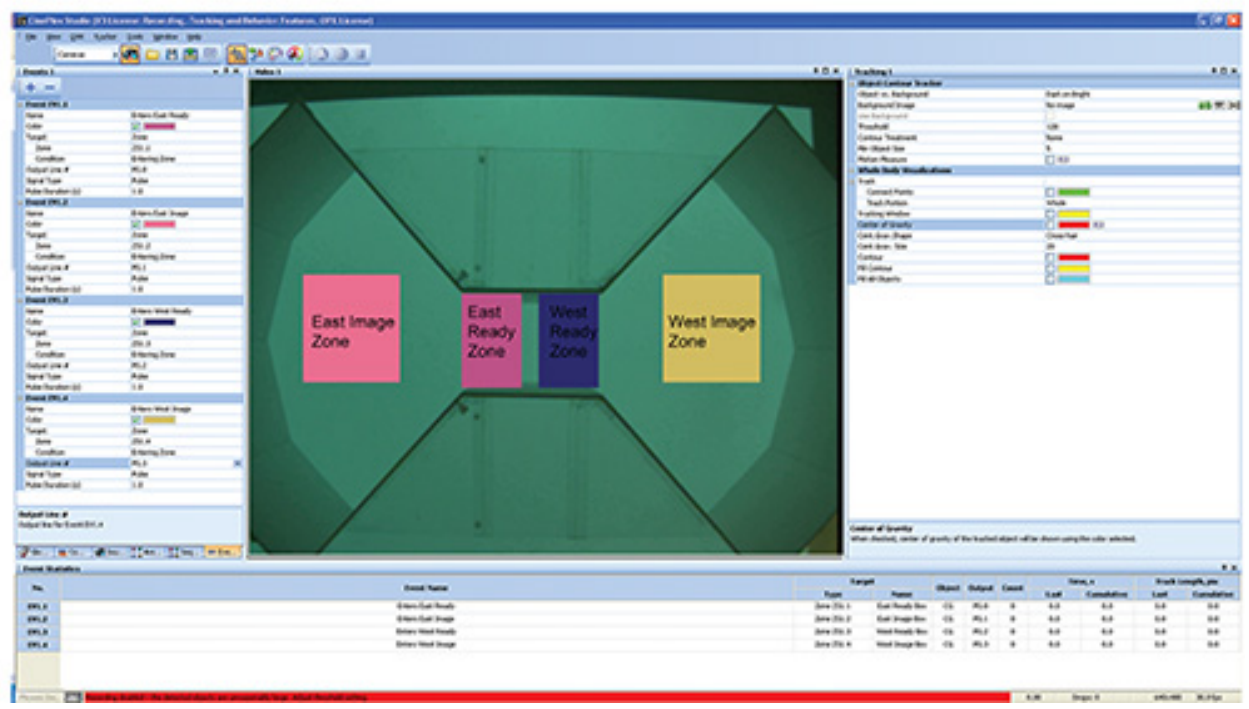

Figure 1. Experimental setup. A. Schematic of the behavioral and control rooms. The Floor Projection Maze is housed in the behavioral room. Rats are monitored via an overhead camera. The control room houses the equipment to control the task and to collect neural data. B Dimensions of the bowtie maze. C. Screen capture of CinePlex Studio. Zones are defined by the user. Logical events are sent as CinePlex digital outputs to the behavioral control system (Med Associates) to monitor the progress of the rat. Click here to view larger image. 
A. Early shaping

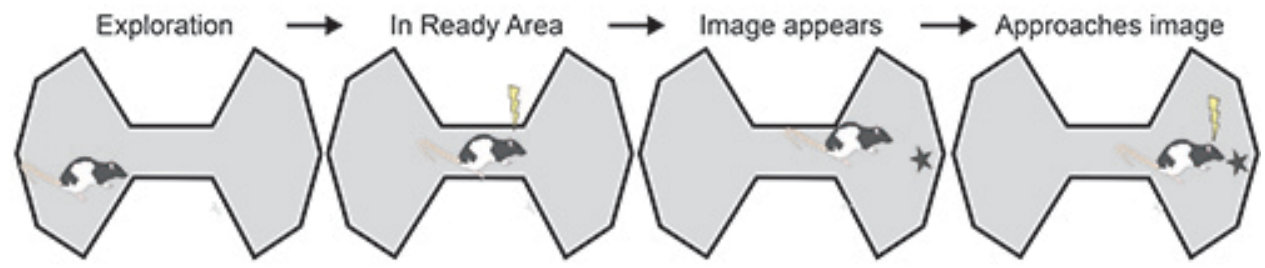

B. Intermediate shaping

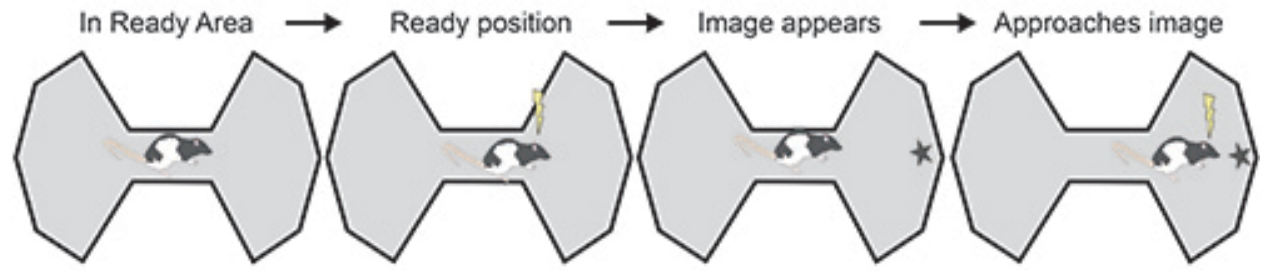

C. Late shaping
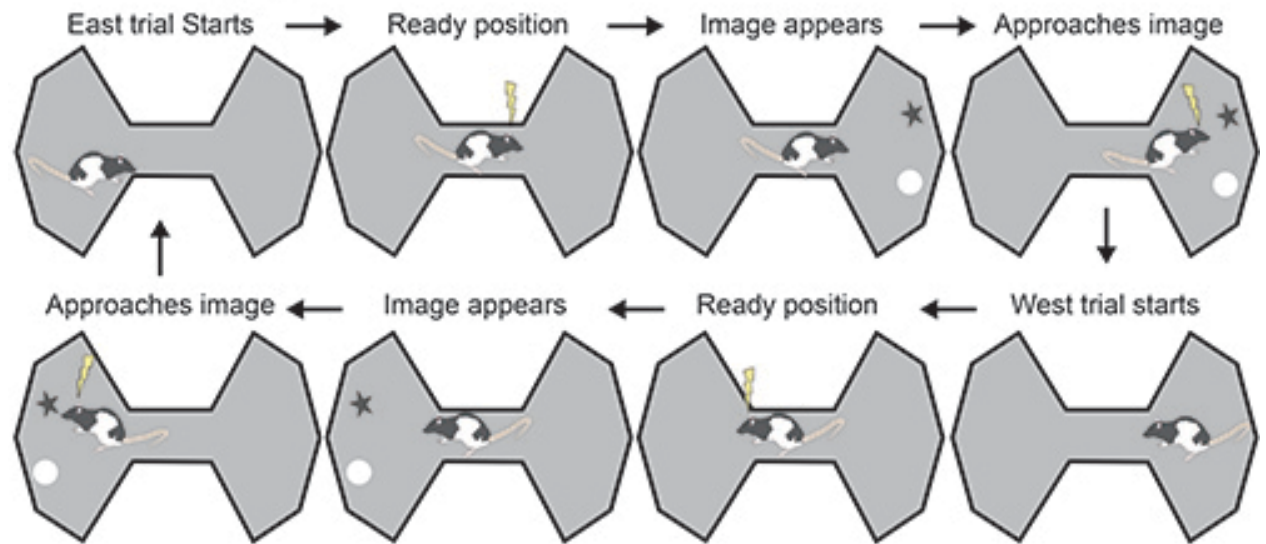

Figure 2. Schematic of the shaping steps. A. In Early shaping the rat receives ICS for entering the Ready Area, and entering East and West Image Areas. The aim is to train the rat to associate these areas with ICS reward. B. Intermediate shaping focuses on training the rats to maintain a stationary 'ready position' in the Ready Area. The images are presented only after maintaining a successful 'ready position'. Rats continue to receive ICS for approaching the image in the Image area. C. At Late shaping, the rat is successfully maintaining a stationary 'ready position'. Training is task specific, and the rat is trained to learn specific rules to perform a given task. Lightning indicates ICS delivery. Click here to view larger image. 


\section{A. Visual biconditional discrimination task}
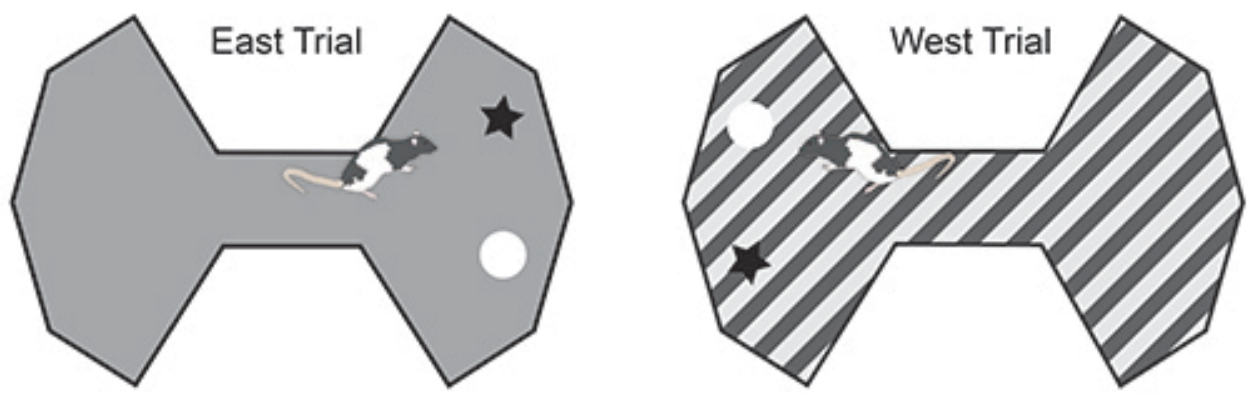

\section{B. Visuospatial attention task}
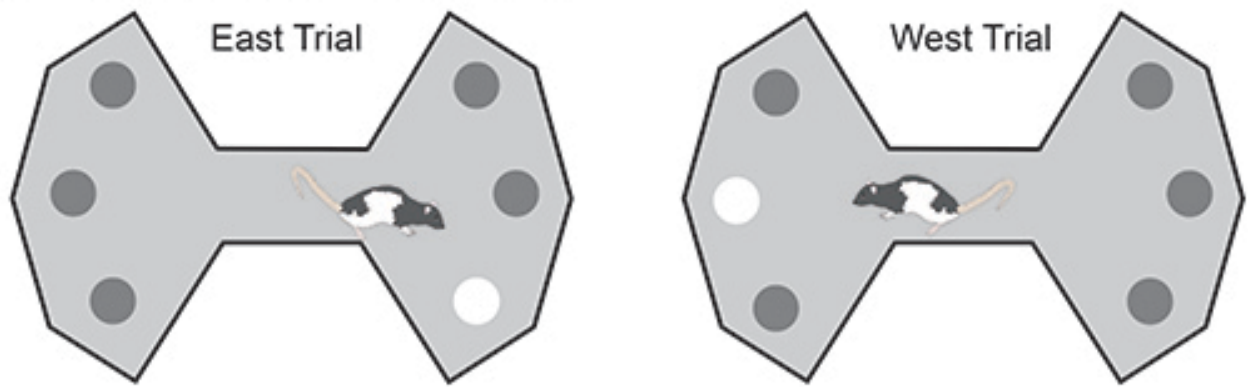

Figure 3. A. Schematic of the vBCD task. When the floor is grey, the black star is rewarded; when the floor is striped, the white circle is rewarded. B. Schematic of the VSA task. Grey circles indicate the target locations in the Image areas. The target stimulus is a brief (500 msec) illumination (white) of one of the circles. The rats are rewarded for approaching the correct target location. Click here to view larger image.

\section{A. Early shaping}

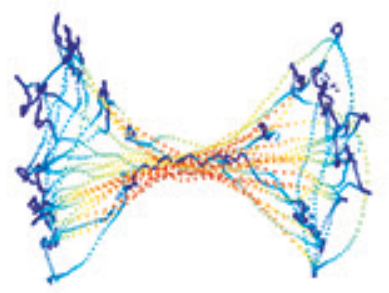

B. Intermediate shaping

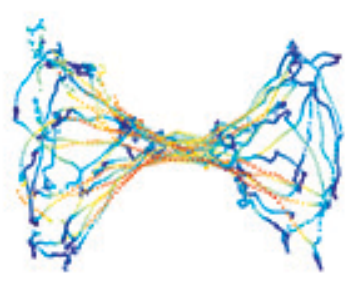

C. Late shaping

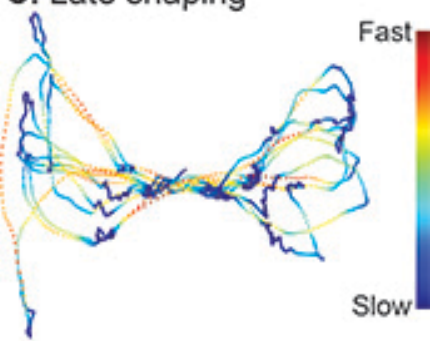

Figure 4. Example paths during shaping. Position data from two minute segments of a rat performing at different shaping steps. Speed of the animal is represented by the heat map. Cold colors represent slowest speeds and hot colors represent fastest speeds. A. During Early shaping rats explore and rear up on the outer walls of the East and West image areas and do not stop in the Ready Area. B. A stereotyped path starts to emerge in Intermediate shaping. Paths form a loop towards the Image areas back to the Ready Area. Rats begin to successfully maintain a 'ready position' in the Ready Area. C. When rats progress onto Late shaping, rats maintain stationary 'ready positions' and the paths of the rats are more stereotyped. Click here to view larger image. 

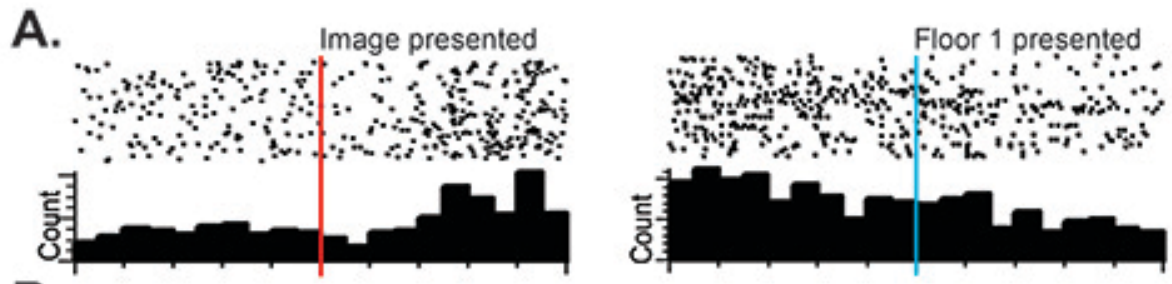

B.
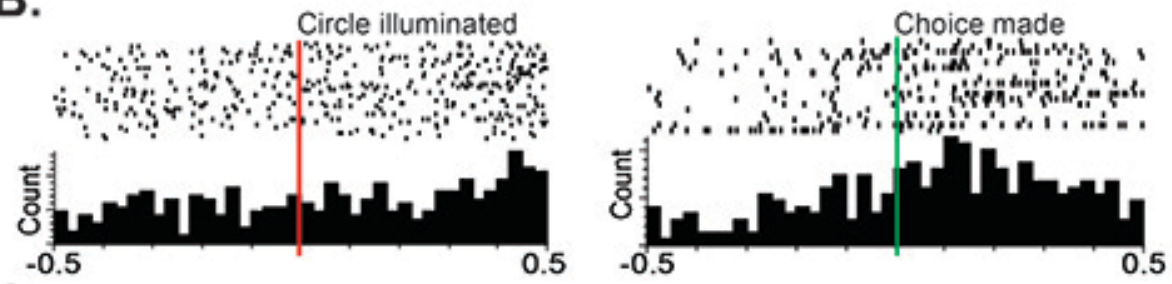

C.

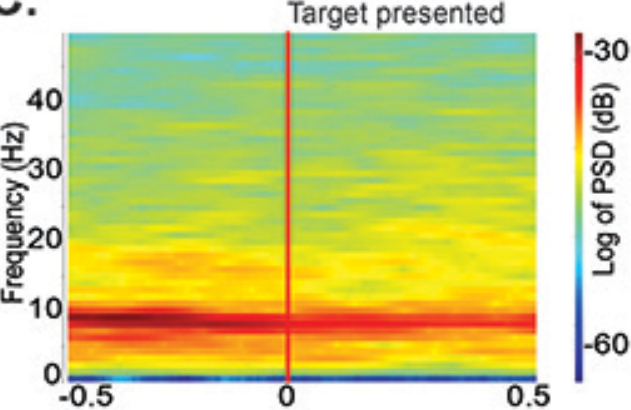

Figure 5. Examples of perievent related analyses. A. Cells recorded from the postrhinal cortex during the vBCD task with different responses to the presentation of the target image (left), and the presentation of the floor (right). B. Cells recorded from the posterior parietal cortex during the VSA task with different responses to the illumination of the circle (left). Increased firing after choosing the spatial location in which the target circle illuminated (right). C. Spectrogram showing strong theta power in the posterior parietal cortex during the 'ready position'. The vertical red line indicates the end of successful 'ready position' and the presentation of the images. The vertical blue line indicates the presentation of Floor 1 at trial start in the VBCD task. The vertical green line indicates that a choice was registered in the VSA task. Click here to view larger image.

\section{Discussion}

We formally describe protocols to train rats on fully automated complex visual cognitive tasks on the Floor Projection Maze. The Floor Projection Maze has been used successfully to establish neural correlates of object-location conjunctions and error signals in postrhinal cortex during performance on a 2-choice visual discrimination task ${ }^{1}$.

The behavioral shaping protocol is designed to be flexible so that it can be adapted to suit an individual rat's learning rate. Custom code written for the behavioral system allows the experimenter to control the probability of automated ICS delivery at critical steps during training; e.g. training the 'ready position'. The code should also allow manual delivery of ICS using a programmable button box during. The aim of Early shaping is to establish effective ICS stimulation for reward delivery to reinforce behavior. When possible we recommend implanting stimulating electrodes bilaterally into MFB in case of ineffective stimulation in one hemisphere. Our experience, however, has been that nearly all implanted ICS electrodes have been functional to deliver a rewarding stimulus. The Intermediate shaping stage is critical for training the rat to maintain a 'ready position' in the Ready Area. In the 'ready position', the rat should be stationary with its nose pointing towards the Image Areas where the choice images will be presented. The 'ready position' controls when the images appear, the direction from which the rat approaches the images and the distance from which the rat views the images. We recommend tracking LEDs mounted on the rat's headstage to monitor the direction in which the rat's head is pointing for better control of behavior and more precise control of target image presentation. Manual delivery of ICS can be issued to train rats to maintain the appropriate 'ready position'. When the rat is maintaining satisfactory 'ready positions' in each trial, training in Late shaping is task specific. In Late shaping rats are trained to learn specific rules to perform a given task.

We use ICS as our method of reward delivery as this method provides instant feedback to the rat resulting in faster learning during behavioral shaping, and more completed trials in a session when collecting neural data in the automated task. ICS delivery, however, results in electrical artifacts in the neural recordings, and can be problematic for analyzing continuous LFP or multiunit data. The interval around the electrical artifact can be removed offline from the neural recording data file prior to continuous data analysis. Stereotypical artifacts from ICS in high pass filtered spike data can be easily removed offline without affecting single spike analyses. Future optimization of protocols for the Floor Projection Maze includes using optogenetic tools to deliver internal reward to minimize and eliminate artifacts associated with electrical stimulation.

The Floor Projection Maze is optimized for the visual capabilities of rats and is thus better suited for visually guided behavioral tasks. It should be noted that direct comparisons cannot be made between experimental paradigms in the Floor Projection Maze and operant chambers because of different experimental protocols employed and different definitions of criterion for successful acquisition of a given task. We have previously shown, however, that rats acquired a simple 2-choice visual discrimination task in $\sim 50$ trials in the Floor Projection Maze ${ }^{2}$ compared with $>300$ 
trials in other studies where images were presented vertically in operant chambers ${ }^{3,10}$. The VSA task was modeled after the classical 5-choice serial reaction task ${ }^{13}$ (5-CSRT) designed to assess visual attentional processes. Typically the 5-CSRT is carried out in an operant chamber and requires the animal to make a nose poke in the appropriate nose-poke hole indicated by a light flash; here the VSA task is carried out in an open arena where animals have to remain stationary at a specific location in the arena, direct their attention to the target locations and wait for the light stimulus to appear. In our experience, rats required $\sim 3,200$ trials to reach criterion ( $75 \%$ on two consecutive days) in the 5 -CSRT ${ }^{14}$ compared $^{-}$ with $\sim 800$ trials in the VSA task in the Floor Projection Maze.

Another benefit of behavioral tasks performed on the Floor Projection Maze is that tasks are carried out in a large open arena, which exploits the natural tendency of the rat to explore its environment, as opposed to tasks that require the animal to fix its gaze to a stationary target. A major advantage of utilizing freely moving rats in an open arena in the Floor Projection Maze apparatus is that both allocentric and egocentric spatial information can be collected with visual information. Another advantage of our paradigm is that tasks need not be confined to presentation of static images. Position and features of visual stimuli that are dynamic can be used in future tasks. Using tracking capabilities of the CinePlex Behavioral Research System, tasks can be developed for a more detailed functional understanding of a given brain structure. Combining simultaneous electrophysiological recordings with visually guided cognitive tasks, fundamental questions of how brain activity is related to cognitive processes such as decision-making and visual discrimination can be explored.

Using visually guided tasks, findings from rat studies can be better translated to humans with the ultimate goal of providing therapies for human cognitive diseases.

\section{Disclosures}

Submission fee for this video-article is sponsored by Plexon Inc.

\section{Acknowledgements}

This work was supported by NSF IOS 1146334, NSF EFRI 0937848, DARPA N66001-10-C-2010, and NSF IOS 0522220 to RDB. We thank

Stacie Hyatt at Plexon Inc for assistance in preparing this manuscript.

\section{References}

1. Furtak, S. C., Ahmed, O. J. \& Burwell, R. D. Single neuron activity and theta modulation in postrhinal cortex during visual object discrimination. Neuron. 76, 976-988, doi:10.1016/j.neuron.2012.10.039 (2012)

2. Furtak, S. C. et al. The Floor Projection Maze: A novel behavioral apparatus for presenting visual stimuli to rats. J. Neurosci. Methods. 181, 82-88, doi:10.1016/j.jneumeth.2009.04.023 (2009).

3. Bussey, T. J. et al. The touchscreen cognitive testing method for rodents: how to get the best out of your rat. Learn. Mem. 15, 516-523, doi:10.1101//m.987808 (2008).

4. Bussey, T. J., Muir, J. L., Everitt, B. J. \& Robbins, T. W. Triple dissociation of anterior cingulate, posterior cingulate, and medial frontal cortices on visual discrimination tasks using a touchscreen testing procedure for the rat. Behav. Neurosci. 111, 920-936 (1997).

5. Forwood, S. E., Bartko, S. J., Saksida, L. M. \& Bussey, T. J. Rats spontaneously discriminate purely visual, two-dimensional stimuli in tests of recognition memory and perceptual oddity. Behav. Neurosci. 121, 1032-1042, doi:10.1037/0735-7044.121.5.1032 (2007).

6. Gaffan, E. A. \& Eacott, M. J. A computer-controlled maze environment for testing visual memory in the rat. J. Neurosci. Methods. 60, 23-37 (1995).

7. Keller, J., Strasburger, H., Cerutti, D. T. \& Sabel, B. A. Assessing spatial vision - automated measurement of the contrast-sensitivity function in the hooded rat. J. Neurosci. Methods. 97, 103-110 (2000).

8. Lashley, K. S. The mechanism of vision V The structure and image-forming power of the rat's eye. J. Comp. Psychol. 13, 173-200, doi:Doi 10.1037/H0074901 (1932).

9. Lashley, K. S. The Mechanism of Vision: Xv. Preliminary Studies of the Rat's Capacity for Detail Vision. J. Gen. Psychol. 18, 123-193 (1938).

10. Minini, L. \& Jeffery, K. J. Do rats use shape to solve "shape discriminations"? Learn. Mem. 13, 287-297, doi:10.1101//m.84406 (2006).

11. Milner, P. M. Brain-Stimulation Reward - a Review. Can. J. Psychol. 45, 1-36, doi:Doi 10.1037/H0084275 (1991).

12. Albasser, M. M. et al. New behavioral protocols to extend our knowledge of rodent object recognition memory. Learn. Mem. 17, 407-419, doi:10.1101/lm.1879610 (2010).

13. Bari, A., Dalley, J. W. \& Robbins, T. W. The application of the 5-choice serial reaction time task for the assessment of visual attentional processes and impulse control in rats. Nat. Protoc. 3, 759-767, doi:http://www.nature.com/nprot/journal/v3/n5/suppinfo/nprot.2008.41_S1.html (2008).

14. Agster, K. L. \& Burwell, R. D. Structure and function of the rodent postrhinal cortex: comparisons to other cortical regions. Ph.D thesis, Brown University, Rhode Island, USA (2007). 